\title{
Editorial: Recent Advances in Cardiotoxicity Testing
}

\author{
Tamer M. A. Mohamed ${ }^{1,2 \star}$, Javid Moslehi ${ }^{3}$ and Jonathan Satin ${ }^{4}$ \\ ${ }^{1}$ Department of Medicine, The Institute of Molecular Cardiology, University of Louisville, Louisville, KY, United States, ${ }^{2}$ Department \\ of Medicine, Diabetes and Obesity Center, Envirome Institute, University of Louisville, Louisville, KY, United States, ${ }^{3}$ Department of \\ Cardio-oncology, University of California, San Francisco, San Francisco, CA, United States, ${ }^{4}$ Department of Physiology, \\ University of Kentucky, Lexington, KY, United States
}

Keywords: cancer, cardiooncology, heart, toxicicity, hiPS cardiomycytes, heart slices

Editorial on the Research Topic

Recent Advances in Cardiotoxicity Testing

\section{INTRODUCTION}

Drug induced cardiotoxicity is a major cause of market withdrawal (Onakpoya et al., 2016). In the last decade of the 20th century, eight non-cardiovascular drugs were withdrawn from clinical use because they prolonged the QT interval (Fermini and Fossa, 2003), resulting in ventricular arrhythmias and potentially sudden death. In particular, the last decade has seen an explosion of cancer therapies, which while effective can lead to several cardiovascular toxicities. Both traditional (e.g., anthracyclines and radiation) and targeted (e.g., trastuzumab) therapies can result in cardiovascular complications in a subset of patients (Groarke et al., 2013; Moslehi, 2016). A close collaboration between cardiologists and oncologists (via the emerging field of "cardiooncology") had helped make these complications manageable ensuring that patients can be treated effectively (Campia et al., 2019). An explosion of novel therapies which include newer kinase inhibitors, proteolysis-targeting chimera (PROTAC) and immune-based therapies expand the oncology armamentarium, each drug with its own potential cardiovascular toxicity (Sheng et al., 2016; Fleming et al., 2021). Therefore, there is a growing need for reliable preclinical screening strategies for $\mathrm{CV}$ toxicities associated with emerging breast cancer therapies prior to human clinical trials. The most prevalently used cardiac physiology screening platform are animal models that have limited reliability in mirroring the effects of drugs in human hearts (Liu et al., 2006; Salama and Bett, 2014; Asnani et al., 2021). Additionally, the use of animal models to create a pharmacokinetic profile of drugs is relatively expensive at the early development stage since large amounts of the drugs are used (Guth et al., 2019). Ultimately, the ideal experimental cardiac tissue culture model is the one that demonstrates high sensitivity and specificity towards various therapeutic and pharmacological interventions while accurately replicating the physiology and pathophysiology of the human heart (Wang et al., 2008). The recent move towards the use of human induced pluripotent stem cellderived cardiomyocytes (hiPS-CMs) (Burridge et al., 2016) and human heart slices (Ou et al., 2019; Miller et al., 2020; Ou et al., 2020) in cardiotoxicity testing provided a potential solution to address this issue. In this special issue we have 12 manuscripts, seven review articles and five original research articles summarizing the recent advances in cardiotoxicity testing.

Jaeger et al. used a novel computer simulation to identifying drug response through combining several assessments of the membrane potential, the cytosolic calcium concentration, and the extracellular potential in microphysiological systems. In another effort for in silico modeling of cardiotoxicity, Paci et al., assessed the response of three human stem cell derived cardiomyocytes 
(hSC-CMs) in silico models to simulate drug action, and compare simulation results against in vitro data for 15 drugs. Furthermore, in an effort to create a novel high-throughput platform to detect cardiotoxicity for atrial arrhythmias, Honda et al. (2021) created and validated a high-throughput drug screening system based on hiPS-derived-atrial myocytes. In the same vein, Verkerk et al. optimized a dynamic clamp system to obtain patch-clamp recordings of action potentials from adult primary human atrial myocytes. This technology will be useful in detecting arrhythmogenic cardiotoxins on adult human hearts. The same first author, Dr. Verkerk with other team of collaborators have modeled a prominent cause of arrhythmia in patients with a deficiency in very long-chain acyl-CoA dehydrogenase (VLCAD), an enzyme that is involved in the mitochondrial beta-oxidation of long-chain fatty acids. They generated hiPSC-CMs from VLCAD deficiency patients and they tested the effect of carnitine supplement on attenuating the arrhythmic potential; however, it was not effective.

This special issue includes seven great reviews and perspectives of different topics related to cardiotoxicity testing. Szabo et al. provided a perspective regarding novel anticancer therapeutics to prevent tumorigenesis without cardiotoxic effects highlighting a new family of therapeutics including ERK dimerization inhibitors and BAX allosteric inhibitors. Campana et al. provided a perspective regarding the role of inflammation in cardiotoxicity and how inflammation should be accounted for during drug screening in early stages of drug development. Thomas et al., Keung et al., and Orsolits et al. have provided three comprehensive reviews for the cellular and tissue models derived from hiPS cells and their recent uses to assess cardiotoxicity of cancer therapies.

\section{REFERENCES}

Asnani, A., Moslehi, J. J., Adhikari, B. B., Baik, A. H., Beyer, A. M., de Boer, R. A., et al. (2021). Preclinical Models of Cancer Therapy-Associated Cardiovascular Toxicity: A Scientific Statement from the American Heart Association. Circ. Res. 129, e21-e34. doi:10.1161/RES.0000000000000473

Burridge, P. W., Li, Y. F., Matsa, E., Wu, H., Ong, S. G., Sharma, A., et al. (2016). Human Induced Pluripotent Stem Cell-Derived Cardiomyocytes Recapitulate the Predilection of Breast Cancer Patients to Doxorubicin-Induced Cardiotoxicity. Nat. Med. 22, 547-556. doi:10.1038/nm.4087

Campia, U., Moslehi, J. J., Amiri-Kordestani, L., Barac, A., Beckman, J. A., Chism, D. D., et al. (2019). Cardio-Oncology: Vascular and Metabolic Perspectives: A Scientific Statement from the American Heart Association. Circulation 139, e579-e602. doi:10.1161/CIR.0000000000000641

Fermini, B., and Fossa, A. A. (2003). The Impact of Drug-Induced QT Interval Prolongation on Drug Discovery and Development. Nat. Rev. Drug Discov. 2, 439-447. doi:10.1038/nrd1108

Fleming, M. R., Xiao, L., Jackson, K. D., Beckman, J. A., Barac, A., and Moslehi, J. J. (2021). Vascular Impact of Cancer Therapies: The Case of BTK (Bruton Tyrosine Kinase) Inhibitors. Circ. Res. 128, 1973-1987. doi:10.1161/CIRCRESAHA.121.318259

Groarke, J. D., Cheng, S., and Moslehi, J. (2013). Cancer-drug Discovery and Cardiovascular Surveillance. N. Engl. J. Med. 369, 1779-1781. doi:10.1056/ NEJMp1313140

Guth, B. D., Engwall, M., Eldridge, S., Foley, C. M., Guo, L., Gintant, G., et al. (2019). Considerations for an In Vitro, Cell-Based Testing Platform for Detection of Adverse Drug-Induced Inotropic Effects in Early Drug Development. Part 1: General Considerations for Development of
Furthermore, Tu et al. wrote a mini review regarding the use of hiPS-derived cells as a screening platform for drug-induced vascular toxicity. hiPSC-CM systems are ideal for highthroughput testing and for proof-of-principle evaluations of patient-specific genetic backgrounds or disease mutations; however, the incomplete maturation and lack of multicellularity creates caveats. The use of slice preparations is an exciting advance in test platforms because this native tissue preparation retains phenotypic properties in vitro (Ou et al., 2019; Miller et al., 2020; Ou et al., 2020). Finally, Meki et al. reviewed the use of adult human and pig heart slices in cardiotoxicity testing.

Overall, this special issue provides an overview of the novel platforms used for cardiotoxicity testing. This field of research is evolving rapidly, and it ultimately holds promise for more reliable and sensitive drug and chemical screening.

\section{AUTHOR CONTRIBUTIONS}

All authors listed have made a substantial, direct, and intellectual contribution to the work and approved it for publication.

\section{FUNDING}

TM is supported by NIH grants R01HL147921 and P30GM127607 and American Heart Association grant 16SDG29950012. We also acknowledge the United States Department of Defense for the grant W81XWH-20-1-0419 (JS and TM).

Novel Testing Platforms. Front. Pharmacol. 10, 884. doi:10.3389/ fphar.2019.00884

Honda, Y., Li, J., Hino, A., Tsujimoto, S., and Lee, J. K. (2021). High-Throughput Drug Screening System Based on Human Induced Pluripotent Stem Cell-Derived Atrial Myocytes A Novel Platform to Detect Cardiac Toxicity for Atrial Arrhythmias. Front. Pharmacol. 12, 680618. doi:10.3389/fphar.2021.680618

Liu, W., Ashford, M. W., Chen, J., Watkins, M. P., Williams, T. A., Wickline, S. A., et al. (2006). MR Tagging Demonstrates Quantitative Differences in Regional Ventricular wall Motion in Mice, Rats, and Men. Am. J. Physiol. Heart Circ. Physiol. 291, H2515-H2521. doi:10.1152/ajpheart.01016.2005

Miller, J. M., Meki, M. H., Ou, Q., George, S. A., Gams, A., Abouleisa, R. R. E., et al. (2020). Heart Slice Culture System Reliably Demonstrates Clinical DrugRelated Cardiotoxicity. Toxicol. Appl. Pharmacol. 406, 115213. doi:10.1016/ j.taap.2020.115213

Moslehi, J. J. (2016). Cardiovascular Toxic Effects of Targeted Cancer Therapies. N. Engl. J. Med. 375, 1457-1467. doi:10.1056/NEJMra1100265

Onakpoya, I. J., Heneghan, C. J., and Aronson, J. K. (2016). Post-marketing Withdrawal of 462 Medicinal Products Because of Adverse Drug Reactions: a Systematic Review of the World Literature. BMC Med. 14, 10. doi:10.1186/ s12916-016-0553-2

Ou, Q., Abouleisa, R. R. E., Tang, X. L., Juhardeen, H. R., Meki, M. H., Miller, J. M., et al. (2020). Slicing and Culturing Pig Hearts under Physiological Conditions. J. Vis. Exp. 20 (157). doi:10.3791/60913

Ou, Q., Jacobson, Z., Abouleisa, R. R. E., Tang, X. L., Hindi, S. M., Kumar, A., et al. (2019). Physiological Biomimetic Culture System for Pig and Human Heart Slices. Circ. Res. 125, 628-642. doi:10.1161/CIRCRESAHA.119.314996

Salama, G., and Bett, G. C. (2014). Sex Differences in the Mechanisms Underlying Long QT Syndrome. Am. J. Physiol. Heart Circ. Physiol. 307, H640-H648. doi:10.1152/ajpheart.00864.2013 
Sheng, C. C., Amiri-Kordestani, L., Palmby, T., Force, T., Hong, C. C., Wu, J. C., et al. (2016). 21st Century Cardio-Oncology: Identifying Cardiac Safety Signals in the Era of Personalized Medicine. JACC Basic Transl Sci. 1, 386-398. doi:10.1016/j.jacbts.2016.05.008

Wang, D., Patel, C., Cui, C., and Yan, G. X. (2008). Preclinical Assessment of Drug-Induced Proarrhythmias: Role of the Arterially Perfused Rabbit Left Ventricular Wedge Preparation. Pharmacol. Ther. 119, 141-151. doi:10.1016/j.pharmthera.2008.02.009

Conflict of Interest: TM holds equities in Tenaya Therapeutics.

The remaining authors declare that the research was conducted in the absence of any commercial or financial relationships that could be construed as a potential conflict of interest.
Publisher's Note: All claims expressed in this article are solely those of the authors and do not necessarily represent those of their affiliated organizations, or those of the publisher, the editors and the reviewers. Any product that may be evaluated in this article, or claim that may be made by its manufacturer, is not guaranteed or endorsed by the publisher.

Copyright (c) 2021 Mohamed, Moslehi and Satin. This is an open-access article distributed under the terms of the Creative Commons Attribution License (CC BY).

The use, distribution or reproduction in other forums is permitted, provided the original author(s) and the copyright owner(s) are credited and that the original publication in this journal is cited, in accordance with accepted academic practice. No use, distribution or reproduction is permitted which does not comply with these terms. 